\title{
EGFR-associated pathways involved in traditional Chinese medicine (TCM)-1-induced cell growth inhibition, autophagy and apoptosis in prostate cancer
}

\author{
ZHAOMENG WU ${ }^{1}$, QINGYI ZHU ${ }^{2}$, YU ZHANG ${ }^{1}$, YINGYING YIN ${ }^{1}$,

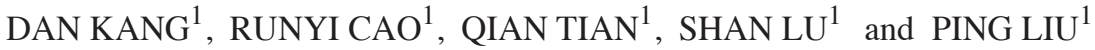 \\ ${ }^{1}$ Jiangsu Key Laboratory for Molecular and Medicine Biotechnology, College of Life Sciences, \\ Nanjing Normal University, Nanjing, Jiangsu 210023; ${ }^{2}$ Laboratory of Molecular Biology, \\ Jiangsu Province Hospital of TCM, Nanjing, Jiangsu 210029, P.R. China
}

Received March 30, 2017; Accepted November 16, 2017

DOI: $10.3892 / \mathrm{mmr} .2018 .8818$

\begin{abstract}
Traditional Chinese medicine (TCM) has the synergistic effect of the combination of a single ingredient and a monomer, and systemic and local therapeutic effects in cancer treatment, through which TCM is able to enhance the curative effect and reduce the side effects. The present study analyzed the effect of TCM-1 (an anti-cancer TCM) on prostate cancer ( $\mathrm{PCa}$ ) cell lines, and studied in detail the mechanism of cell death induced by TCM-1 in vitro and in vivo. From the present results, it was identified for the first time, to the best of our knowledge, that TCM-1 arrested the cell cycle at the G1 phase, decreased cell viability and increased nuclear rupture in a dose-dependent manner; these effects finally resulted in apoptosis in PCa cells. At the molecular level, the data demonstrated that TCM-1 competitively acted on epidermal growth factor receptor (EGFR) with EGF, and suppressed the auto-phosphorylation and activity of EGFR. Inhibition of EGFR further suppressed the downstream phosphatidylinositol 3-kinase (PI3K)/RAC- $\alpha$ serine/threonine-protein kinase (AKT) and RAF proto-oncogene serine/threonine-protein kinase/extracellular signal regulated kinase signaling pathways and resulted in a decrease in the phosphorylated-forkhead box protein O1 (at Ser256, Thr24 and Ser319) expression level, and induced cell growth inhibition and apoptosis by regulating the expression of apoptosis-and cell cycle-associated genes. In addition, TCM-1 markedly inhibited the PI3K/AKT/serine/threonine-protein kinase mTOR signaling pathway and induced cell autophagy
\end{abstract}

Correspondence to: Professor Ping Liu, Jiangsu Key Laboratory for Molecular and Medicine Biotechnology, College of Life Sciences, Nanjing Normal University, 1 Wenyuan Road, Nanjing, Jiangsu 210023, P.R. China

E-mail: 08201@njnu.edu.cn

Key words: traditional Chinese medicine-1, prostate cancer, epidermal growth factor-associated pathway, apoptosis, autophagy by downregulating the phosphorylation of p70S6K and upregulating the levels of Beclin-1 and microtubule-associated protein light chain-3II. In vivo, the TCM-1-treated group exhibited a significant decrease in tumor volume compared with the negative control group in subcutaneous xenograft nude mice by inhibiting EGFR-associated signaling pathways. Therefore, the bio-functions of Chinese medicine TCM-1 in inducing $\mathrm{PCa}$ cell growth inhibition, autophagy and apoptosis suggested that TCM-1 may have clinical potential for the treatment of patients with PCa.

\section{Introduction}

Prostate cancer ( $\mathrm{PCa}$ ) is the second most prevalent type of cancer affecting the health of men in Western countries. According to Surveillance, Epidemiology, and End Results Program (National Institutes of Health, Bethesda, MD, USA) statistics, there were 220,800 novel cases of PCa in the USA in 2015 and 86,380 patients succumbed to PCa (1). Due to the inefficiency of chemotherapy and radiotherapy, therapeutic strategies for this disease are limited and metastatic disease frequently develops, even following potentially curative surgery (2-4). Therefore, it is of great importance to develop novel therapeutics for the treatment of prostate cancer.

Overexpression of epidermal growth factor receptor (EGFR) has been reported in a number of types of solid tumor, including prostate cancer (5). Activation of EGFR induces the phosphorylation and activation of its downstream signaling pathways, including the phosphatidylinositol 3-kinase $(\mathrm{PI} 3 \mathrm{~K}) / \mathrm{RAC}-\alpha$ serine/threonine protein kinase (AKT) and RAF proto-oncogene serine/threonine-protein kinase (Raf)/extracellular signal-regulated kinase (Erk) pathways, and finally leads to cell proliferation (6). Activated AKT disturbs the balance of apoptosis and cell viability by promoting nuclear factor $(\mathrm{NF})-\kappa \mathrm{B}$ and inhibiting the pro-apoptotic transcription factors forkhead box proteins (FOXs) (7-9). Overexpression of FOXs (including FOXO1 in prostate cancer cells) triggers cell cycle arrest and induces cellular apoptosis by increasing the levels of Fas-ligand(Fas-L), tumor necrosis factor ligand superfamily member 10 (TRAIL), and Bcl-2-like protein 11 (Bim) in cells of 
various tissue types (10). As reported, the PI3K/AKT/serine/threonine-protein kinase mTOR (mTOR)/ribosomal protein S6 kinase $\beta-1$ (p70S6K) signaling pathway is the primary pathway that regulates autophagy when cells are exposed to certain conditions, including starvation, oxidative stress and tumor suppression (11). Beclin-1 serves an important role in autophagosome formation and in the crosstalk between autophagy and apoptosis, and the expression of Beclin-1 is regulated by the $\mathrm{PI3K} / \mathrm{Raf} /$ Erk pathway and Bcl-2 (12).

Previous studies have suggested that the occurrence of certain diseases (including cancer) is associated with immune suppression, and a number of traditional Chinese medicines (TCMs) are able to correct the state of immune suppression by improving the immune system and the disease resistance of the human body, there by achieving the purpose of treating diseases $(13,14)$. In the clinical treatment of cancer in a number of Hospitals of TCM, the results demonstrate that TCM has a holistic and a local therapeutic effect, in addition to the synergistic effect of the combination of single ingredients and monomers. Although TCM has the unique advantages of the whole-body and complementary effects that single medicine (Western medicine) does not have in anti-cancer treatment (15), the molecular mechanism of the bio-functions of TCM in the treatment of various cancers remains to be completely elucidated.

The present study investigated the effects of TCM-1 (water-extract from a type of anti-PCa TCM) on prostate cancer cells (including androgen-dependent LNCaP and androgen-independent PC3 cell lines) in vitro and in vivo. The results indicated that TCM-1 was able to decrease cell viability and arrest the cell cycle at the G1 phase, finally resulting in marked autophagy and apoptosis in prostate cancer cells. From the results of the present study, TCM-1 exerteda dose-dependent downregulation of the activity of the EGFR signaling pathway by inhibiting EGF-induced autophosphorylation of EGFR. Inhibition of EGFR activity resulted in restraining its downstream signaling pathways (including the PI3K/AKT and Erk pathways) and then increased the translocation (from cytoplasm to nucleus) and the transcriptional activity of FOXO1; in addition, it upregulated the expression of apoptosis-associated and autophagy-associated proteins and down-regulated cell cycle-associated proteins. Therefore, the Chinese medicine TCM-1 markedly inhibited cell growth and promoted autophagy and apoptosis in prostate cancer cells, suggesting that TCM-1 hada potential clinical value for treatment of prostate cancer in clinic.

\section{Materials and methods}

Cell culture and TCM-1. Prostate cancer cell lines (including LNCaP, CWR22Rv1, PC3 and DU145) and the normal prostate cell line WPMY-1 were cultured in RPMI 1640 medium (Wisent Biotechnology Co., Ltd., Nanjing, China), supplemented with $10 \%$ fetal bovine serum (Gibco; Thermo Fisher Scientific, Inc., Waltham, MA, USA), penicillin (100 U/ml) and streptomycin (100 mg/ml) (Sigma-Aldrich; Merck KGaA, Darmstadt, Germany), in a humidified $5 \% \mathrm{CO}_{2}$ atmosphere at $37^{\circ} \mathrm{C}$.

TCM-1, the water-extract from a compound TCM, was provided by Jiangsu Province Hospital of TCM (Nanjing,
China) and contained $1 \mathrm{~g} / \mathrm{ml}$ pharmaceutical raw materials, which were composed of Astragalusmembranaceus, radix Rehmanniaepreparata, Curcuma zedoaria, Rhizoma wenyujin concisum, Rhizoma paridis chinensis, Polygonum perfoliatum L., Radix Glycyrrhizae, and Ficus pumila L. For the in vivo experiments, TCM-1 was diluted in 1XPBS for intragastric administration.

Antibodies and reagents. Antibodies against poly (ADP-ribose) polymerase 1 (PARP-1; cat. no. sc-56196), caspase3 (cat. no. sc-271028), Cyclin D1 (cat. no. sc-246), E3 ubiquitin-protein ligase XIAP (cat. no. sc-55552), AKT (cat. no. sc-55523), prostate specific antigen (PSA; cat. no. sc-7316), phospho (p)-AKT (Ser-473; cat. no. sc-271964), Histone H3 (cat. no. sc-10809), FOXO1 (cat. no. sc-514610), Bim (cat. no. sc-11425), cyclin-dependent kinase inhibitor 1 (p21; cat. no sc-817), p53 (cat. no. sc-126), p27 (cat. no. sc-53906) EGFR (cat. no. sc-101), p-EGFR (Tyr1173; cat. no. sc-57545) and $\beta$-actin (cat. no. sc-47778) were purchased from Santa Cruz Biotechnology, Inc. (Dallas, TX, USA). Antibodies against Erk (cat. no. 5013), p-Erk1/2 (T202/Y204; cat. no. 9106), p-AKT (Thr308; cat. no. 4050) and GAPDH (cat. no. 2118) were purchased from Biogot Technology Co., Ltd. (Nanjing, China). Antibodies against p-FOXO1 (Ser256/Thr24/Ser319; cat nos. 84192, 9464 and 2486, respectively), p-PI3K p85 (Tyr458)/p55 (Tyr199) (cat. no. 4228) were purchased from Cell Signaling Technology, Inc. (Danvers, MA, USA). Antibodies against caspase8/p18 (cat. no. 66093-1), caspase9/p35/p10 (cat. no. 10380-1), Bcl-2 (cat. no. 60178-1), Bax (cat. no. 50599-2), Raf-1 (cat. no. 51140-1), microtubule-associated proteins $1 \mathrm{~A} / 1 \mathrm{~B}$ light chain $3 \mathrm{~B}$ (LC3)-I/II (cat. no. 18725-1), Beclin-1 (cat. no. 11306-1), mTOR (cat. no. 20657-1), p70S6K (cat. no. 14485-1), PI3K p85 (cat. no. 21739-1) and proliferation marker protein Ki-67 (cat. no. 27309-1) were purchased from ProteinTech Group, Inc. (Chicago, IL, USA). Antibodies for Fas ligand (FasL; cat. no. AF0157), p-Raf-1 (Ser338; cat. no. AF3065), p-transcription factor p65 (p65; Ser536; cat. no. AF2006), p65 (cat. no. BF0382), p-dual specificity mitogen-activated protein kinase kinase (MEK)1/2(Ser217/Ser221) (cat. no. AF8035), MEK1/2 (cat. no. AF6385), p-mTOR (Ser2448; cat. no. AF3308) and p-p70S6K (Thr389/Thr412; cat. no. AF3228) were purchased from Affinity Biosciences (Cambridge, UK). Horseradish peroxidase conjugated secondary antibodies, including rabbit IgG (cat. no. HAF008) and mouse IgG (HAF007), were purchased from Bio-Techne China Co. Ltd., R \& D Systems (Shanghai, China). Assay kits for radioimmunoprecipitation assay (RIPA), MTT and Cell Counting Kit-8 (CCK-8) were purchased from Beyotime Institute of Biotechnology (Haimen, China). The Nuclear/Cytosol Fractionation kit was purchased from AmyJet Scientific (Wuhan, China). DAPI was purchased from Beijing Solarbio Science \& Technology Co., Ltd. (Beijing, China), dissolved in 1X PBS and used at a concentration of $20 \mu \mathrm{g} / \mathrm{ml}$. hEGF was purchased from Merck KGaA (Darmstadt, Germany). hEGF was directly added to cell culture medium at the concentration of 0,20 and $30 \mathrm{ng} / \mathrm{ml}$ for $30 \mathrm{~min}$ in cell incubator with $5 \% \mathrm{CO}_{2}$ at $37^{\circ} \mathrm{C}$. Other chemicals were all purchased from Sigma-Aldrich (Merck KGaA).

DAPI staining assay for nuclear condensation rupture. For the DAPI staining assay, LNCaP and PC3 cells were cultured in 
12-well plates and incubated with increasing doses of TCM-1 $(0,2,5$ and $10 \mathrm{mg} / \mathrm{ml})$ for $24 \mathrm{~h}$. LNCaP and PC3 cells were washed with 1X PBS briefly and fixed in $4 \%$ formaldehyde for $15 \mathrm{~min}$, and washed three times with $1 \mathrm{X}$ PBS and then permeabilized in $0.2 \%$ Triton X-100 for $15 \mathrm{~min}$. Cells were then stained with $6 \mu \mathrm{l}$ DAPI $(1 \mathrm{mg} / \mathrm{ml})$ adding $300 \mu \mathrm{l} 1 \mathrm{X}$ PBS at room temperature for $8 \mathrm{~min}$ and were photographed with a fluorescence microscope (Nikon IX-71; Nikon Corporation, Tokyo, Japan).

Flow cytometry assay for cellular apoptosis and the cell cycle. LNCaP and PC3 cells were seeded in 6-well plates $\left(0.5 \times 10^{5}-1 \times 10^{5} /\right.$ well $)$ and treated with different concentrations of TCM-1 $(0,2,5$ and $10 \mathrm{mg} / \mathrm{ml})$ for $24 \mathrm{~h}$, and cells were collected for flow cytometric analysis (cellular apoptosis assay) using an annexin V-fluorescein isothiocyanate (FITC) apoptosis detection kit (Beyotime Institute of Biotechnology, Haimen, China). In detail, cells were harvested and resuspended in $1 \mathrm{X}$ binding buffer (contained in the kit). Annexin V-FITC and propidium iodide (PI) were added to the cells, according to manufacturer's protocol, for 10-20 min at room temperature while protected from light. Flow cytometry analysis for cell apoptosis was performed using a flow cytometer and analyzed by ModFit LT (version 2.0; Verity Software House, Inc., Topsham, ME, USA), and a minimum of 30,000 events was collected for each sample.

For the cell cycle assay, LNCaP and PC 3 cells were seeded in 6 -well plates $\left(0.5 \times 10^{5}-1 \times 10^{5} /\right.$ well $)$ and treated with the concentrations of TCM-1 (0, 2, 5 and $10 \mathrm{mg} / \mathrm{ml})$ for $24 \mathrm{~h}$. LNCaP and PC3 cells were collected and fixed with $70 \%$ ethanol overnight at $4^{\circ} \mathrm{C}$. Subsequently, the samples were treated with $100 \mu \mathrm{l}$ RNase A (10 mg/ml) for $30 \mathrm{~min}$ at $37^{\circ} \mathrm{C}$, stained with $200 \mu \mathrm{l} \mathrm{PI}$ $(10 \mathrm{mg} / \mathrm{ml})$ for $0.5 \mathrm{~h}$ at $4^{\circ} \mathrm{C}$, and $800 \mu \mathrm{l} 1 \mathrm{X}$ PBS was added. Flow cytometry analysis was performed using a flow cytometer and a minimum of 20,000 events was collected for each sample. The raw collected data were analyzed by ModFit LT (version 2.0; Verity Software House, Inc.) to determine cell cycle distribution.

MTT assay and CCK-8 assay for cell viability. MTT and CCK-8 assays were performed to assess the cell viability. Cells were seeded in a 96-well plate at a density of $1 \times 10^{4}$ cells/well overnight and treated with different concentrations of TCM-1 $(0,2,5$ and $10 \mathrm{mg} / \mathrm{ml})$ for $24 \mathrm{~h}$.

For the MTT assay, the culture medium was removed and fresh medium $(100 \mu \mathrm{l})$ was added with $10 \mu \mathrm{l}$ MTT $(5 \mathrm{mg} / \mathrm{ml})$. The plate was incubated at $37^{\circ} \mathrm{C}$ for $4 \mathrm{~h}$ in the dark. The medium was removed again, and $100 \mu \mathrm{l}$ dimethyl sulfoxide was added to each well. The absorbance at $570 \mathrm{~nm}$ was measured using a microplate reader (Thermo Fisher Scientific, Inc.).

For the CCK-8 assay, the culture medium was removed and fresh medium $(100 \mu \mathrm{l})$ was added with CCK-8 solution $(5 \mu \mathrm{l})$. The plate was incubated for $4 \mathrm{~h}$ at $37^{\circ} \mathrm{C}$ in the dark. Absorbance was measured using a microplate reader (Thermo Fisher Scientific, Inc.) at $450 \mathrm{~nm}$.

The measured optical density values were converted into cell viability according to the manufacturer's protocol.

Western blot analysis and chromatin immunoprecipitation (ChIP) assay. Following $24 \mathrm{~h}$ of treatment with TCM-1 (0, 2, 5 and $10 \mathrm{mg} / \mathrm{ml}), \mathrm{LNCaP}$ and PC3 cells were harvested and lysed by the addition of $120 \mu \mathrm{l}$ RIPA buffer [Tris-HCl, $\mathrm{Ph}$ 7.6;1\% NP-40; $0.1 \%$ sodium deoxycholate; 0.1\% SDS; $150 \mathrm{mM} \mathrm{NaCl} ; 1 \mathrm{mM}$ EGTA; $1 \mathrm{mM}$ phenylmethylsulfonyl fluoride; $1 \%$ Triton X-100; and Roche complete protease inhibitor cocktail (Roche Diagnostics, Basel, Switzerland)]. Tumor tissues were cut in to small fragments. RIPA buffer was added and the fragments were ground with a manual tissue grinder. Following centrifugation $\left(12,000 \mathrm{x} \mathrm{g}, 4^{\circ} \mathrm{C}, 10 \mathrm{~min}\right)$ of cell lysates and tumor tissue lysates, the supernatants were collected and the total protein concentration was quantified using Bradford reagent (Bio-Rad Laboratories, Inc., Hercules, CA, USA). Equal amounts of total protein $(15 \mu \mathrm{g})$ were separated by $10 \%$ SDS-PAGE and transferred to a polyvinylidene difluoride membrane (Bio-Rad Laboratories, Inc., Hercules, CA, USA). Following $1 \mathrm{~h}$ blocking with 5\% skimmed milk at room temperature, the transferred membranes were blotted using primary antibodies (all 1:1,000 dilution) overnight at $4^{\circ} \mathrm{C}$, and corresponding peroxidase-labeled secondary antibodies (all 1:10,000 dilution) at room temperature for $1 \mathrm{~h}$. Bands were detected using an Enhanced Chemiluminescence Detection kit (GE Healthcare, Chicago, IL, USA).

For the ChIP assay, LNCaP cells were treated with TCM-1 (0 and $5 \mathrm{mg} / \mathrm{ml})$. At $12 \mathrm{~h}$, cells were cross-linked with $1 \%$ formaldehyde for $10 \mathrm{~min}$ at $25^{\circ} \mathrm{C}$. Cells were harvested, sonicated $(300 \mathrm{~W}$, sonication $2 \mathrm{sec}$, interval $20 \mathrm{sec}$, 20 cycles in an ice bath) and the soluble chromatin fragments (200-500 bp) were incubated with $2 \mu \mathrm{g}$ rabbit IgG or FOXO1 antibodies. DNA-protein immune complexes were eluted and reverse cross-linked, and DNA was extracted using spin filter columns. The presence of the p21 promoter domain in immunoprecipitated DNA was identified using the following primers: Forward primer, 5'-GGTGTCTAGGTGCTCCAG GT-3'; and reverse primer, 5'-GCACTCTCCAGGAGGACA CA-3'. The amplified p21 promoter region was analyzed via $1 \%$ agarose/ethidium bromide gel electrophoresis. In the control samples, the primary antibodies were replaced with non-immune rabbit IgG.

In vivo efficacy of treatment with TCM-1 in the prostate tumor xenograft mouse model. A total of 32 nude mice (male, 5-week old) were purchased from the Model Animal Research Center of Nanjing University, Nanjing, China and raised in SPF level animal room at room temperature with normal diet and drinking water, 40-70\% humidity, $0.03 \% \mathrm{CO}_{2}$ and $12 \mathrm{~h}$ ligh/dark cycle. PC3 cells $\left(1 \times 10^{6}\right)$ were suspended in PBS $(100 \mu \mathrm{l})$ and injected subcutaneously into the flanks of each animal. Mice were randomly divided into four groups (8 mice/group), including a negative control group (1X PBS), positive control group [5-fluorouracil (FU); $30 \mathrm{mg} / \mathrm{kg}$ ], a low-dose TCM-1 group (0.5 g/kg), and a high-dose TCM-1 group $(2.0 \mathrm{~g} / \mathrm{kg})$. When the tumors grew to $24-30 \mathrm{~mm}^{3}$, mice were intragastrically administered negative/positive control reagents and low-/high-doses of TCM-1 every 2 days for 6 weeks. The tumor length/width and body weight were measured at the end of each treatment week. The tumor volume was calculated as: Volume $\left(\mathrm{mm}^{3}\right)=\left(\right.$ length $\mathrm{x}$ width $\left.{ }^{2}\right) / 2$. At the end of the experiments, the mice were sacrificed on the $42 \mathrm{nd}$ day and tumors were dissected, weighed and snap-frozen with liquid nitrogen for further western blot (as described above) and immunohistochemical analyses. All animal experiments 
in the present study were approved (permit no. NL-129-02) by the Ethics Committee of Jiangsu Province Hospital of TCM, Nanjing, China.

Immunohistochemistry (IHC) assay. Tumors were fixed in $10 \%$ buffered formalin $\left(4^{\circ} \mathrm{C}\right.$, overnight), embedded in paraffin and sectioned to $5 \mu \mathrm{m}$ in size. Each tissue section was deparaffinized and rehydrated with xylene and gradient concentrations of ethanol. Tissue sections were boiled in EDTA for $15 \mathrm{~min}$, quenched with $0.3 \%$ hydrogen peroxide solution for $10 \mathrm{~min}$ at room temperature and blocked with bovine serum albumin (Gibco; Thermo Fisher Scientific Inc.) in PBS for $60 \mathrm{~min}$ at $30^{\circ} \mathrm{C}$. Slides were subsequently incubated with specific primary antibodies (all 1:50 dilution) overnight at $4^{\circ} \mathrm{C}$. Sections were counterstained with hematoxylin (10 $\mathrm{min}$, at room temperature). Antibody binding was detected with an EnVision Detection kit, Peroxidase/Diaminobenzidine, Rabbit/Mouse (Gene Tech Biotechnology Co., Ltd., Shanghai, China). The expression levels of specific proteins were observed and photographed under a microscope at a magnification of $\mathrm{x} 400$ (CTR 6000; Leica Microsystems GmbH, Wetzlar, Germany), and the proliferation index was expressed as the percentage of positive cells relative to the total number of cells in a given area.

Statistical analysis. All data are expressed as the mean \pm standard deviation. Comparisons between groups were made by one-way analysis of variance followed by Dunnett's test, using SPSS software (version 19.0; IBM Corp., Armonk, $\mathrm{NY}, \mathrm{USA}$ ). $\mathrm{P}<0.05$ was considered to indicate a statistically significant difference. All experiments were replicated three times.

\section{Results}

TCM-1 induces morphological changes and inhibits cell viability in prostate cancer cells. To assess the effect of TCM-1 on prostate cell lines, WPMY-1 and PCa cells (including androgen-dependent LNCaP and CWR22Rv1, and androgen-independent PC3 and DU145) were cultured and treated with different concentrations of TCM-1 (0, 2, 5 and $10 \mathrm{mg} / \mathrm{ml}$ ) for $24 \mathrm{~h}$. Cellular morphological alterations and cell viability were photographed using a microscope and determined by MTT and CCK- 8 assays. From the data, it was observed that TCM-1 induced morphological alterations in PCa cells in a concentration-dependent manner; cells appeared shrunken and rounded, and certain cells were lysed, whereas no distinct alterations were noted in the normal prostate cell WPMY-1 even when treated with $10 \mathrm{mg} / \mathrm{ml}$ of TCM-1 (Fig. 1A). In addition, the MTT and CCK-8 assays demonstrated that the proliferation and viability of PCa cells were significantly decreased by treatment with TCM-1 in a concentration-dependent manner, whereas the proliferation and viability of WPMY-1 cells were almost unaffected by treatment TCM-1 (Fig. 1B and C). These results indicated that TCM-1 suppressed cell growth and proliferation, in addition to decreasing cell viability, particularly in prostate cancer cells.

In order to address the molecular mechanism of the effect of TCM-1 on PCa cells, the androgen-dependent LNCaP cell line and the androgen-independent $\mathrm{PC} 3$ cell line were selected as the cellular models for subsequent experiments.
TCM-1 induces cell cycle arrest and apoptosis in PCa cells. To identify TCM-1-induced cell cycle arrest and apoptosis in PCa cells, $\mathrm{LNCaP}$ and PC 3 cells were cultured and treated with different concentrations of TCM-1 $(0,2,5$ and $10 \mathrm{mg} / \mathrm{ml})$ for $24 \mathrm{~h}$. Cells were treated for flow cytometry analysis to assess cell cycle arrest and apoptosis and for the DAPI staining assay to determine PCa cellular apoptosis (Fig. 2). As presented in Fig. 2A, apoptosis in PCa cells (including LNCaP and PC3 cells) was markedly induced by treatment with TCM-1. The degree of cellular apoptosis (including early apoptosis and late apoptosis) was dependent on the concentration of TCM-1. As presented in Fig. 2C, the cell cycle of $\mathrm{LNCaP}$ and PC3 cells was arrested at the G1 phase following treatment with TCM-1, and the degree of G1 phase arrest was dependent on the concentration of TCM-1. The number of cells in the G1 phase was increased, while cells in the $\mathrm{S}$ and $\mathrm{G} 2 / \mathrm{M}$ phases were decreased following treatment with TCM-1. In addition, the DAPI staining assay demonstrated that TCM-1 induced the formation of apoptotic bodies and nuclear shriveling in $\mathrm{LNCaP}$ and PC3 cells. The number of cells exhibiting shriveled nuclei and apoptotic bodies was increased with the increasing concentration of TCM-1 (Fig. 2B).

TCM-1 inhibits the PI3K/AKT signaling pathway and activates FOXO1 by downregulating the phosphorylation levels of PI3K, AKT and FOXO1 in PCa cells. $\mathrm{LNCaP}$ and PC3 cells were seeded in 6-well plates and treated with different concentrations of TCM-1, as indicated in Fig. 3. At $24 \mathrm{~h}$, cells were harvested for western-blot analysis. The results demonstrated that treatment with TCM-1 in PCa cells decreased p-AKT (including Ser473 and Thr308) and p-PI3K expression levels, while no apparent alterations in the total AKT and PI3K protein expression levels were noted. The decreasing p-AKT and p-PI3K levels presented a TCM-1 concentration-dependent manner (Fig. 3A and B). In addition, the phosphorylation level of P-65 (p-p65Ser536), a subunit of NF- $\mathrm{KB}$ which as a downstream target of PI3K/AKT pathway, was also decreased by treated TCM-1 in a concentration dependent manner (Fig. 3A and B). These results indicated that TCM-1 inhibited the activity of NF- $\kappa B$ by inhibiting the PI3K/AKT signaling pathway.

FOXO1 serves an important role in a number of cellular physiological processes, including cell growth, cell proliferation and apoptosis in prostate cancer cells; in addition, FOXO1 is an important downstream target of the PI3K/AKT pathway (16). Phosphorylation of FOXO1 at Ser256/Thr24/Ser319 by the PI3K/AKT pathway induces the translocation of FOXO1 from the nucleus to the cytoplasm (17). The results of the present study demonstrated that the phosphorylation levels at Ser256/Thr24/Ser319, and not the total protein level of FOXO1, were decreased in $\mathrm{LNCaP}$ and $\mathrm{PC} 3$ cells following treatment with TCM-1; this decrease was dependent on the treatment concentration of TCM-1 (Fig. 3C and D). In addition, these data illustrated that the nuclear FOXO1 levels increased and the cytoplasmic FOXO1 levels decreased following treatment with TCM-1, and these trends were dependent on the treatment concentration of TCM-1 (Fig. 3E and F). The ChIP assay data demonstrated that TCM-1 increased the levels of FOXO1 binding to the promoter of the p21 gene (Fig. 3G). In addition, treatment with TCM-1 in LNCaP cells downregulated the levels of PSA, which is a downstream target gene of the 

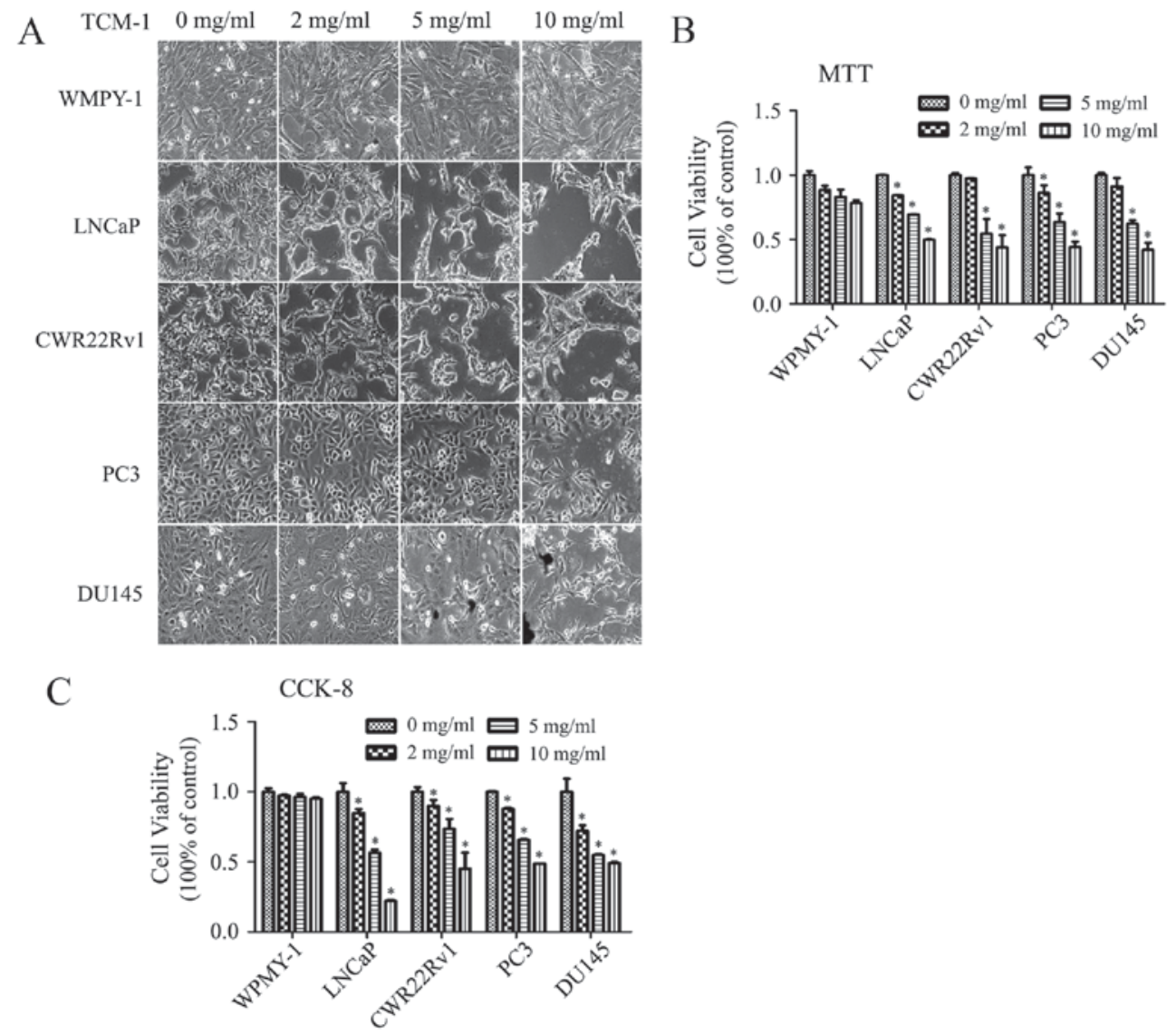

Figure 1. TCM-1 induces cellular morphological alterations and inhibits cell viability in prostate cancer cells. (A) WPMY-1, LNCaP, CWR22Rv1, PC3 and DU145 cells were seeded in a 12-well plate and incubated for $24 \mathrm{~h}$ with different concentrations of TCM-1 (0,2, $5 \mathrm{and} 10 \mathrm{mg} / \mathrm{ml})$. Morphological alterations in the cells were observed and photographed with a microscope at x40magnification. In order to study the effects of TCM-1 on proliferation and viability, cells were treated with different concentrations of TCM-1 (0,2, 5 and $10 \mathrm{mg} / \mathrm{ml})$ for $24 \mathrm{~h}$. Cell viability was measured via (B) MTT and (C) CCK-8 assays. Experiments were performed three times. The results are expressed as the mean \pm standard deviation $(n=6)$ and as a percentage of the vehicle-treated control." $\mathrm{P}<0.05$ vs. respective $0 \mathrm{mg} / \mathrm{ml}$ group. TCM-1, traditional Chinese medicine 1; CCK-8, Cell Counting Kit-8.

androgen receptor (AR) (Fig. 3H), suggesting that AR maybe involved in TCM-1-induced cell growth inhibition and apoptosis in androgen-dependent PCa cell lines.

These results indicated that TCM-1 inhibited the activity of the PI3K/AKT signaling pathway by decreasing the phosphorylation levels of PI3K and AKT, and additionally decreased the phosphorylation level of FOXO1 (Ser256/Thr24/Ser319), resulting in the translocation of FOXO1 proteins into the nucleus from the cytoplasm and increasing the transcriptional activity of FOXO1.

TCM-1 induces the activation of intrinsic and extrinsic apoptotic pathways in a cellular tumor antigen p53 (p53)-independent manner in PCa cells. It has been reported that FOXO1 is a tumor suppressor, and that overactivation of FOXO1 may induce cancer cell apoptosis by stimulating the expression of death receptor ligands, including FasL and TRAIL, in addition to inducing the expression of a number of pro-apoptotic members of the apoptosis regulator Bcl-2 family of mitochondria-targeting proteins (10). From the present experimental data, it was observed that the expression of the FOXO1-targeted pro-apoptotic Bim and FasL proteins was markedly increased following treatment with TCM-1, in a concentration-dependent manner (Fig. 4A and B). It is known that FasL is a death ligand that is able to trigger the extrinsic apoptotic pathway through binding to its receptor Fas, expressed on the majority of cancer cells; Bim is able to trigger the intrinsic apoptotic pathway (10). In the present study, the experimental data demonstrated that TCM-1 decreased the expression levels of anti-apoptotic proteins (including Bcl-2, XIAP and survivin) (Fig. 4C and D), and increased the expression levels of pro-apoptotic proteins [including Bax, cleaved (c)-PARP-1, c-caspase 9, c-caspase 8 and c-caspase 3] (Fig. 4E and F) in LNCaP and PC3 cells. These results indicated that treatment with TCM-1 in PCa cells activated the intrinsic and extrinsic apoptotic pathways simultaneously by increasing the expression of Bim and FasL via the activation of FOXO1. In addition, p53 is a well-known tumor suppressor, which is known to cause cell cycle arrest, autophagy and apoptosis in a number of types of cancer cells (18). From the experimental results, it was observed that the protein level of p53 was almost unaltered following treatment with TCM-1 in LNCaP cells (no expression of p53 was detected in PC3 cells) (Fig. 4E and F).

These data suggested that TCM-1 inhibited the activity of the PI3K/AKT pathway and induced the translocation of FOXO1 into the nucleus from the cytoplasm, activating the intrinsic and extrinsic apoptosis pathways (caspase-dependent) 
A
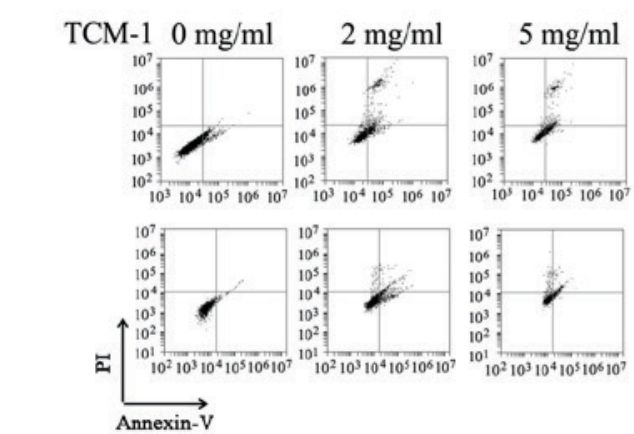

$\mathrm{C}$

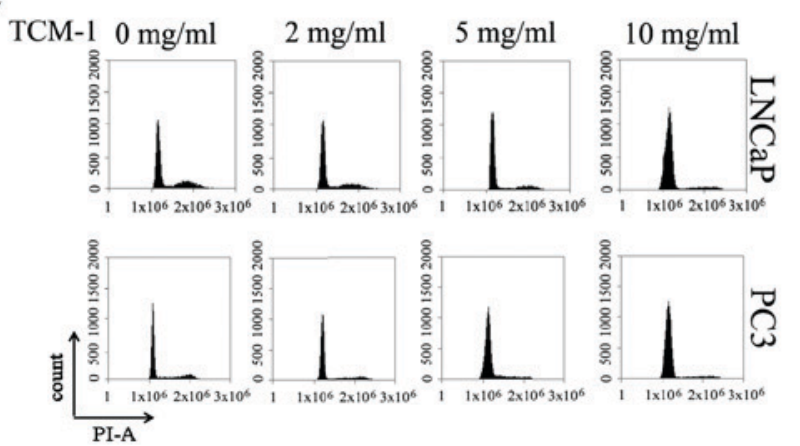

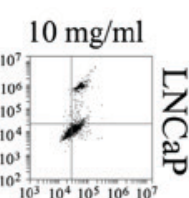
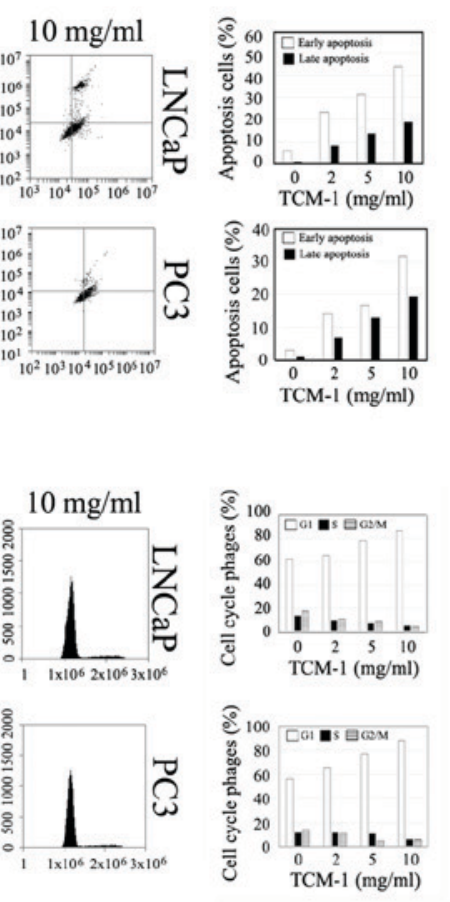

$\mathrm{B}$

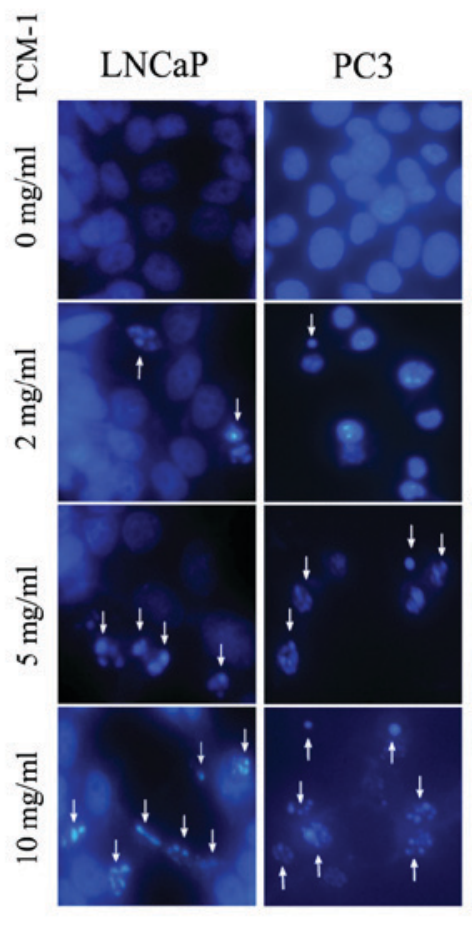

Figure 2. TCM-1 induces cellular apoptosis and cell cycle arrest in LNCaP and PC3cells. (A) LNCaP and PC3 cells were cultured and treated with different concentrations of TCM-1 (0, 2, 5 and $10 \mathrm{mg} / \mathrm{ml})$ for $24 \mathrm{~h}$. Cells were harvested and subjected to a cellular apoptosis assay using annexin V-FITC and PI double-staining flow cytometry analysis. Percentages of cellular apoptosis (including early and late apoptosis) were quantified. (B) Cells were treated with TCM-1 $(0,2,5$ and $10 \mathrm{mg} / \mathrm{ml})$ for $24 \mathrm{~h}$, and the nuclear morphology was observed and photographed following DAPI staining under a microscope, using a blue filter with x40 magnification. Arrows indicate fragmented nuclei. (C) To assess the effect of TCM-1 on the cell cycle, LNCaP and PC3 cells were cultured in 12-well plates overnight and treated with different concentrations of TCM-1 (0,2, 5 and $10 \mathrm{mg} / \mathrm{ml})$ for $24 \mathrm{~h}$. The cells were harvested and stained with PI to analyze the cell cycle by flow cytometric analysis. Percentages of cell cycle phases were quantified. TCM-1, traditional Chinese medicine 1 ; FITC, fluorescein isothiocyanate; PI, propidium iodide.

in prostate cancer cells. TCM-1-induced PCa apoptosis was observed to be p53-independent.

TCM-1 inhibits the activity of the Raf/MEK/Erk signaling pathway, resulting in downregulation of Cyclin D1, and upregulation of 21 and cyclin-dependent kinase inhibitor $1 B$ (p27), in PCa cells. As previously reported, inhibition of the Raf/MEK/Erk signaling pathway downregulates Cyclin D1 and upregulates $\mathrm{p} 21 / \mathrm{p} 27$ by activating the transcription factor FOXO1, resulting in cell cycle arrest at the G1 phase $(10,19)$. In order to determine whether the Raf/MEK/Erk signaling pathway was involved in cell cycle arrest at the $\mathrm{G} 1$ phase in TCM-1-treated PCa cells, LNCaP and PC3 cells were cultured and treated with different concentrations of TCM-1, as indicated in Fig. 5, for $24 \mathrm{~h}$. Cells were harvested for western blot analysis to assess the expression levels of cell cycle-associated proteins, including Cyclin D1, p27 and p21. As presented in Fig. 5A and B, treatment with TCM-1 in LNCaP and PC3 cells markedly decreased the protein level of Cyclin D1, whereas it increased the protein expression levels of p21 and p27. These results indicated that TCM-1 induced cell cycle arrest at the G1 phase in LNCaP and PC3 cells, and were consistent with the results of the flow cytometry analysis presented in Fig. 2B. The Raf/MEK/Erk signaling pathway has been demonstrated to serve an important role in promoting the cell cycle, cell growth and cell proliferation (6). In order to identify that
TCM-1 induced PCa cell cycle arrest and growth inhibition by inhibiting the activity of the Raf/MEK/Erk signaling pathway, LNCaP and PC3 cells were cultured and treated with different concentrations of TCM-1, as indicated in Fig. 5C and D, for $24 \mathrm{~h}$. Cells were subsequently harvested and lysed for western blot analysis. The experimental data demonstrated that TCM-1 decreased the phosphorylation level of Raf-1 (Ser338) in a concentration-dependent manner, while no apparent effect on the total protein expression level of Raf-1 in LNCaP and PC3 cells was observed (Fig. 5C and D). Inactivation of Raf-1 further resulted in a decrease in the phosphorylation levels of MEK1/2 (Ser217/Ser221) and Erk1/2 (Thr202/Tyr204), while no effect on the total protein expression levels of MEK1/2 and Erk1/2 in LNCaP and PC3 cells was observed (Fig. 5C and D). These results suggested that TCM-1 induced cell cycle arrest and cell growth inhibition by inactivating the Raf/MEK/Erk signaling pathway.

TCM-1 induces cellular autophagy by dysregulating the mTOR/p70S6K pathway in PCa cells. mTOR, an important factor involved in cellular autophagy, is a downstream target of and is regulated by the PI3K/AKT pathway, originating from starvation, growth factors and cellular suppressors (20). In order to examine whether mTOR-associated cellular autophagy was involved in TCM-1-induced cell growth inhibition and cellular apoptosis, LNCaP and PC3 cells were cultured in 6-well 
A

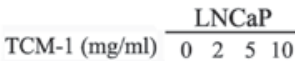
IB: p-PI3K/P85 (Y458) $\boldsymbol{r}$. है IB: PI3K/P85 - IB: p-AKT (S473) - - IB: p-AKT (T308) IB: AKT IB: p-p65(Ser536) IB: $\beta$-actin $-0-$

C

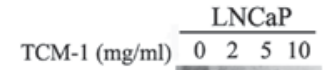
IB: p-FOXO1 (S256) IB: p-FOXO1 (T24) 을 IB: p-FOXO1 (S319) IB: FOXO1 IB: $\beta$-actin

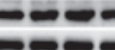

E

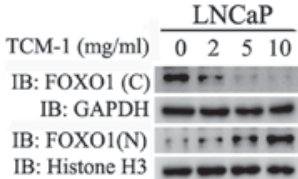

G

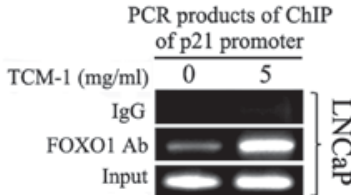

B

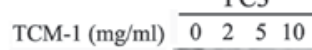
IB: p-PI3K/P85 (Y458) 그 IB: PI3K/P85 $-0-$ IB: p-AKT (S473) $\geq-\ldots$ IB: p-AKT (T308) IB: AKT $-\infty \cdot \cdots$

IB: p-p65(Ser536) IB: $655=--$

IB: $\beta$-actin

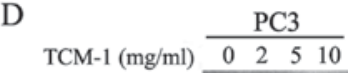
IB: p-FOXO1 (S256) - IB: p-FOXO1 (T24) IB: p-FOXO1 (S319) IB: FOXO1 IB: $\beta$-acti

F

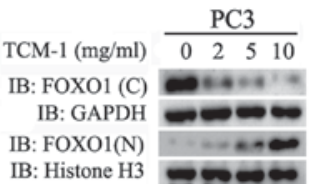

$\mathrm{H}$

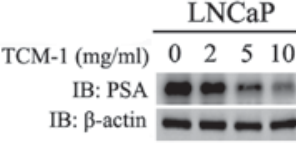

Figure 3. TCM-1 inhibits the PI3K/AKT signaling pathway and activates FOXO1 by downregulating the phosphorylation levels of PI3K, AKT and FOXO1 in LNCaP and PC3cells. (A) LNCaP cells and (B) PC3 cells were cultured in 6-well plates and treated with different concentrations of TCM-1 $(0,2,5$ and $10 \mathrm{mg} / \mathrm{ml})$. At $24 \mathrm{~h}$, cells were harvested and lysed for western blot analysis to assess the protein expression levels of $\mathrm{p}-\mathrm{PI} 3 \mathrm{~K} / \mathrm{p} 85$ (Y458), PI3K/p85, p-AKT(S473), p-AKT(T308), AKT, p-p65(S536), p65 and $\beta$-actin (loading control). (C) LNCaP cells and (D) PC3 cells were cultured and treated as above. At $24 \mathrm{~h}$, cells were harvested and lysed for western blot analysis to assess the protein expression levels of p-FOXO1 (Ser256/Thr24/Ser319), FOXO1, and $\beta$-actin (loading control). (E) LNCaP cells and (F) PC3 cells were harvested for the isolation of subcellular fractions of the nucleus and cytoplasm using a Nuclear/Cytosol Fractionation kit. Protein expression levels of FOXO1 in the nucleus and cytoplasm were separately analyzed by western-blot. (G) LNCaP cells were treated with 0 and $5 \mathrm{mg} / \mathrm{ml}$ TCM-1 for $12 \mathrm{~h}$ and cells were treated for the ChIP assay. The polymerase chain reaction products were assayed by agarose gel electrophoresis. (H) LNCaP and PC3 cells were cultured in 6-well plates and treated with different concentrations of TCM-1 (0, 2, 5 and $10 \mathrm{mg} / \mathrm{ml})$. At $24 \mathrm{~h}$, cells were harvested and lysed for western blot analysis to assess the protein expression levels of PSA and $\beta$-actin (loading control). p, phosphorylated; PI3K, phosphatidylinositol 3-kinase AKT, RAC- $\alpha$ serine/threonine-protein kinase; FOXO1, forkhead box protein O1; p65, transcription factor p65; PSA, puromycin-sensitive aminopeptidase IgG, immunoglobulin G; IB, immunoblotting; TCM-1, traditional Chinese medicine 1; ChIP, chromatin immunoprecipitation; p21, cyclin-dependent kinase inhibitor 1; C, cytoplasmic; $\mathrm{N}$, nuclear; $\mathrm{Ab}$, antibody.

plates and treated with different concentrations of TCM-1, as indicated in Fig. 6, for $24 \mathrm{~h}$. Cells were harvested and lysed for western blot analysis. The experimental data demonstratedthat treatment with TCM-1 in PCa cells decreased the phosphorylation level of mTOR (Ser2448) and resulted in inhibition of mTOR activity, with no marked effect on the total protein expression level of mTOR (Fig. 6A and B). Inhibition of mTOR activity further resulted in activity inhibition of p70S6K by decreasing the phosphorylation level of p70S6K (Thr389/Thr412) in LNCaP and PC3 cells. The expression level of LC3-II was increased with the inhibition of p70S6K,

A

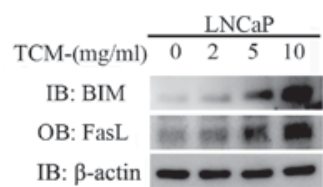

B

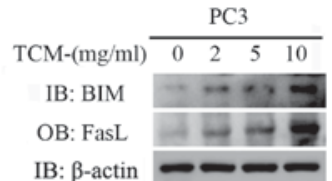

C

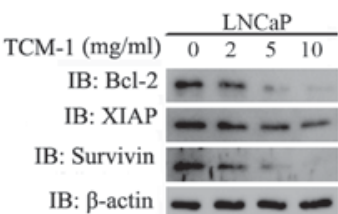

D

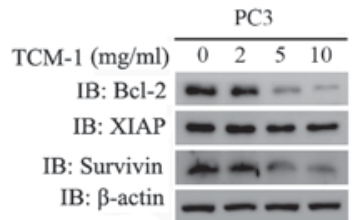

E

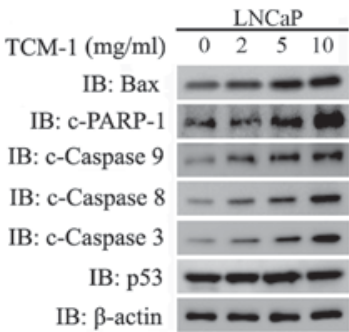

F

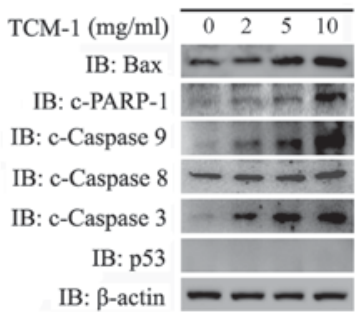

Figure 4. TCM-1 induces the activation of the intrinsic and extrinsic apoptotic pathways in a p53-independent manner in LNCaP and PC3 cells (A) LNCaP and (B) PC3 cells were incubated overnight and treated with different concentrations of TCM-1 $(0,2,5$ and $10 \mathrm{mg} / \mathrm{ml})$ for $24 \mathrm{~h}$. The cells were harvested for western blot analysis to assess the protein levels of Bim, Fas-L and $\beta$-actin (loading control). (C) LNCaP and (D) PC3 cells, treated as described above, were lysed for western blot analysis to detect the protein levels ofBcl-2, XIAP, Survivin and $\beta$-actin (loading control). Whole cell lysates of (E) LNCaP and (F) PC3 cells, treated as above, were subjected to western blot analysis to determine the protein levels of Bax, caspase- $9 /-8 /-3$, PARP-1, p53 and $\beta$-actin (loading control). TCM-1, traditional Chinese medicine 1; Bim, Bcl-2-like protein 11; Fas-L, Fas ligand; Bcl-2, apoptosis regulator Bcl-2; XIAP, E3 ubiquitin-protein ligase XIAP; PARP-1, poly (ADP ribose) polymerase 1; c, cleaved; Bax, apoptosis regulator BAX; p53, cellular tumor antigen p53; IB, immunoblotting.

while the level of LC3-I was decreased (Fig. 6A and B). All the alterations were TCM-1 concentration-dependent, as presented in Fig. 6. Additionally, Beclin-1, a protein which is negatively correlated with Bcl-2, was significantly increased by treatment with TCM-1 in a concentration-dependent manner, via the TCM-1-mediated decrease in the protein expression level of Bcl-2 (Figs. 4C and D, 6A and B).

Considering the inhibition of the PI3K/AKT signaling pathway by TCM-1 (Fig. 3A and B), the results of the present study demonstrated that the TCM-1-induced cellular autophagy via inhibition of them TOR/p70S6K signaling pathway in $\mathrm{LNCaP}$ and $\mathrm{PC} 3$ cells was mediated via inhibition of the activity of the PI3K/AKT signaling pathway, suggesting that cellular autophagy may be involved in TCM-1-induced cell growth inhibition and apoptosis in prostate cancer cells.

TCM-1 targets EGFR and competitively acts on EGFR with $E G F$, resulting in inhibition of the auto-phosphorylation of EGFR. It has been reported that the PI3K/AKT and Raf/MEK/Erk signaling pathways are two important downstream signaling pathways of the EGF/EGFR signaling pathway (21). Over-activation of the EGF/EGFR pathway frequently occurs in a number of types of cancer, and has been demonstrated to contribute to cancer cell 
A

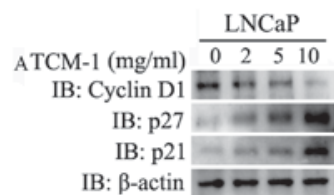

$\mathrm{C}$

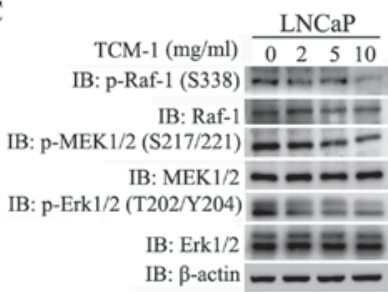

B

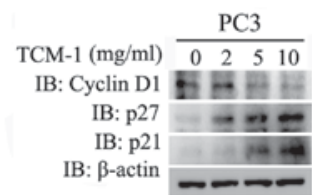

$\mathrm{D}$

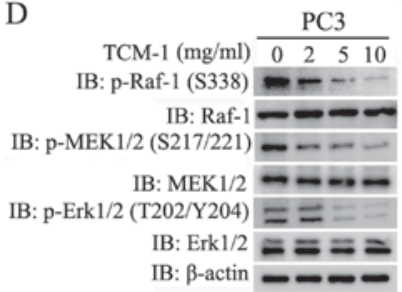

Figure 5. TCM-1 inhibits the activity of the Raf/MEK/Erk signaling pathway, resulting in downregulation of Cyclin D1, and upregulation of p21 and p27, in prostate cancer cells. (A) LNCaP and (B) PC3 cells were cultured overnight and treated with different concentrations of TCM-1 $(0,2,5$ and $10 \mathrm{mg} / \mathrm{ml})$ for $24 \mathrm{~h}$. Cells were harvested for western blot analysis to assess the protein levels of Cyclin D1, p27, p21 and $\beta$-actin (loading control). (C) LNCaP and (D) PC3 cells were treated as described above for $24 \mathrm{~h}$. Cells were harvested and lysed for western blot analysis to determine the protein expression levels of p-Raf (Ser338), Raf, p-MEK1/2(S217/S221), MEK1/2, p-Erk1/2(T202/Y204), Erk $1 / 2$ and $\beta$-actin (loading control). TCM-1, traditional Chinese medicine 1; Raf, RAF proto-oncogene serine/threonine-protein kinase; $\mathrm{p} 21$, cyclin-dependent kinase inhibitor 1; p27, cyclin-dependent kinase inhibitor 1B; MEK, dual specificity mitogen-activated protein kinase kinase; Erk, extracellular signal-regulated kinase; IB, immunoblotting; p, phosphorylated.

A

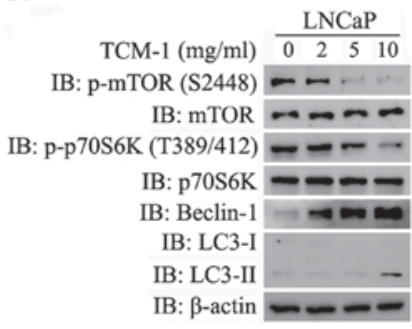

B

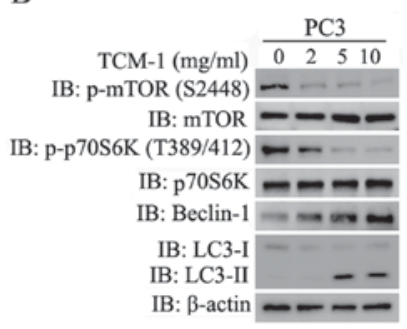

Figure 6. Expression of autophagy-associated proteins in $\mathrm{LNCaP}$ and $\mathrm{PC} 3$ cells following treatment with different doses of TCM-1. (A) LNCaP and (B) PC3 cells were incubated and treated with increasing doses of TCM-1 $(0,2,5$ and $10 \mathrm{mg} / \mathrm{ml})$ for $24 \mathrm{~h}$. Cells were harvested for western blot analysis to assess the protein expression levels of p-mTOR(S2448), mTOR, p-p70S6K(T389/412), p70S6K, Beclin-1, LC3-I, LC3-II and $\beta$-actin (loading control). TCM-1, traditional Chinese medicine 1; p, phosphorylated; mTOR, serine/threonine-protein kinase mTOR; p70S6K, ribosomal protein S6 kinase $\beta-1$; LC3, microtubule-associated proteins 1A/1B light chain 3B.

proliferation, tumor vascularization and metastasis $(22,23)$. In order to determine whether the TCM-1-induced inhibition of the PI3K/AKT and Raf/MEK/Erk signaling pathways occurred via the TCM-1-induced inhibition of the EGF/EGFR signaling pathway, LNCaP and PC3 cells were cultured and treated with different concentration of EGF and/or TCM-1 (5 mg/ml), or different concentrations of TCM-1 and/or EGF (30 ng/ml), as indicated in Fig. 7. From the experimental data, it was revealed that treatment with TCM-1 markedly decreased the auto-phosphorylation levels of EGFR (p-EGFR Tyr1173) and did not alter the total protein expression level of EGFR in LNCaP and PC3 cells. The decrease in p-EGFR (Tyr1173) expression levels was dependent on the treatment concentration of TCM-1 (Fig. 7A and B).
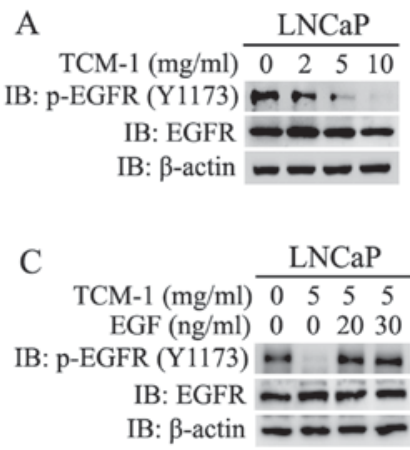

$\mathrm{E}$ \begin{tabular}{rlcccc} 
& \multicolumn{4}{c}{ LNCaP } \\
\cline { 2 - 5 } TCM-1 (mg/ml) & 0 & 0 & 5 & 10 \\
EGF $(\mathrm{ng} / \mathrm{ml})$ & 0 & 30 & 30 & 30
\end{tabular}

IB: p-EGFR (Y1173) IB: EGFR IB: $\beta$-actin

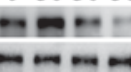

$-\infty$

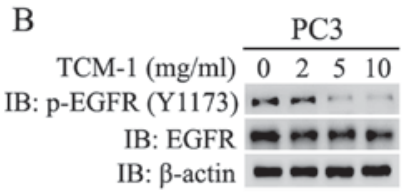

$\mathrm{D}$ TCM-1 (mg/ml) \begin{tabular}{llll} 
& & \multicolumn{3}{ll}{$\mathrm{PC} 3$} & \\
\cline { 2 - 5 } & 5 & 5 & 5
\end{tabular} $\mathrm{EGF}(\mathrm{ng} / \mathrm{ml}) \quad 0 \quad 0 \quad 2030$ IB: p-EGFR (Y1173) IB: $\beta$-actin

\begin{tabular}{|c|c|}
\hline $\mathrm{F}$ & PC3 \\
\hline TCM-1 (mg/ml) & $\begin{array}{llll}0 & 0 & 5 & 10\end{array}$ \\
\hline $\mathrm{EGF}(\mathrm{ng} / \mathrm{ml})$ & $\begin{array}{lllll}0 & 30 & 30 & 30\end{array}$ \\
\hline : p-EGFR (Y1173) & $n=-n$ \\
\hline IB: EGFR & 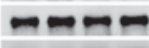 \\
\hline
\end{tabular}
IB: EGFR $\leadsto-\bullet-$

Figure 7. Expression levels of p-EGFR and EGFR in LNCaP and PC3 cells treated with TCM-1 or EGF, and co-treated with TCM-1 and EGF. (A) LNCaP and (B) PC3 cells were incubated and treated with increasing doses of TCM-1 $(0,2,5$ and $10 \mathrm{mg} / \mathrm{ml})$ for $24 \mathrm{~h}$. Cells were harvested for western blot analysis to assess the protein expression levels of p-EGFR, EGFR and $\beta$-actin (loading control). (C) LNCaP and (D) PC3 cells were incubated and pre-treated with $5 \mathrm{mg} / \mathrm{ml} \mathrm{TCM}-1$, and stimulated with different concentrations of EGF (20 or $30 \mu \mathrm{g} / \mathrm{ml}$ ) for $10 \mathrm{~min}$. Cells were harvested and lysed for western blotting to assessthe protein expression levels of p-EGFR (Y1173), EGFR and $\beta$-actin (loading control). (E) LNCaP and (F) PC3 cells were pre-treated with the indicated concentrations $(0,5$ and $10 \mathrm{mg} / \mathrm{ml})$ of TCM- 1 for $24 \mathrm{~h}$ and stimulated with EGF (30 $\mu \mathrm{g} / \mathrm{ml})$ for $10 \mathrm{~min}$. Cells were harvested to determine the expression of p-EGFR (Y1173) and EGFR by western blot analysis. EGFR, epidermal growth factor receptor; TCM-1, traditional Chinese medicine 1; p, phosphorylated.

When LNCaP and PC3 cells were treated or co-treated with TCM-1 $(5 \mathrm{mg} / \mathrm{ml})$ and $\operatorname{EGF}(0,20$ and $30 \mathrm{ng} / \mathrm{ml})$, it was observed that the TCM-1-induced inhibition of the auto-phosphorylation of EGFR was rescued by co-treatment with EGF (Fig. 7C and D). It was additionally demonstrated that the EGF-induced enhancement of the auto-phosphorylation of EGFR was impaired by co-treatment with TCM-1 in PCa cells (LNCaP and PC3) (Fig. 7E and F).

These results demonstrated that TCM-1 downregulated the auto-phosphorylation and activation of the membrane receptor EGFR, and additionally impaired the EGF-induced activation of EGFR. These results indicated that the TCM-1-induced activity inhibition of EGFR and its downstream signaling pathways, including the EGFR/PI3K/AKT and EGFR/PI3K/Erk pathways, maybe due to TCM-1 competitively acting on EGFR with EGF.

TCM-1 suppresses the growth of human PCa cells in vivo by inhibiting EGFR-associated signaling pathways and inducing cellular autophagy and apoptosis. In order to validate the findings from the in vitro studies and to test the efficacy of TCM-1 for prostate cancer therapy, an in vivo study was performed using a PC3 cell tumor xenograft model in nude mice (Fig. 8). PC3 cells were implanted subcutaneously into the right or left flank of nude mice and tumors were allowed to grow. When the tumor volume reached $20-24 \mathrm{~mm}^{3}$, mice were treated with 1x PBS (as a negative control), TCM-1 (0.5 g/ kg as a low dose and $2 \mathrm{~g} / \mathrm{kg}$ as a high dose) and 5-FU (as a positive control; 
$30 \mathrm{mg} / \mathrm{kg}$ ) by oral gavage every 2 days for 6 weeks. Tumor growth and mouse weights were monitored at the end of each treatment week. Following treatment for 6 weeks, mice were sacrificed and the subcutaneous tumors were resected, weighed, photographed and measured immediately. The tumor samples were sectioned and subjected to an IHC assay and western blotting. Compared with the negative control group, tumor growth in vivo was inhibited by low-dose TCM-1, high-dose TCM-1 and the 5-FU positive control (Fig. 8B). Dunnett's test analysis demonstrated that significant differences existed between the high-dose group or positive control group and the negative control group $(\mathrm{P}=0.0353 ; \mathrm{P}=0.0414)$, although a significant difference was not observed between the low-dose group and the negative control group $(\mathrm{P}=0.1230)$. Treatment with TCM-1 revealed a time-dependent inhibition of prostate tumor growth in vivo (tumors in the high-dose group were markedly smaller compared with those of the low-dose group), and an apparent reduction in tumor volume was observed during the 2nd week in the TCM-1-treated group (Fig. 8B), while no signs of toxicity were observed during the treatment period, as indicated by a stable bodyweight (Fig. 8A). The data in Fig. 8B and $\mathrm{C}$ demonstrated that the inhibition of tumor growth by high-dose TCM-1 was almost equal to that induced by 5-FU (positive control). At the end of experiment, the tumor size in the mice was markedly reduced by the treatment with TCM-1 in a dose-dependent manner (Fig. 8C). In addition, the protein expression levels of Ki-67 (marker of cell proliferation), p-EGFR (Tyr1173) and p-AKT (Ser473) were assessed via an IHC assay in PC3 tumor tissues from different groups. The results demonstrated that the treatment with TCM-1 markedly decreased the protein expression levels of $\mathrm{Ki}-67$, and the phosphorylation levels of EGFR (Tyr1173) and AKT (Ser473) in tumor tissues (Fig. 8E). In addition, the western blotting data from the PC3 tumor tissues demonstrated that the expression levels of p-Erk1/2, p-FOXO1 (Ser256) and CyclinD1 were all downregulated by treatment with TCM-1, whereas the protein expression levels of c-caspase 3, c-PARP-1 and LC3-II were upregulated by treatment with TCM-1 (Fig. 8D). Therefore, the in vivo experiments suggested that treatment with TCM-1 induced PCa cellular autophagy and apoptosis by inhibiting the EGFR/PI3K/AKT and EGFR/PI3K/Erk signaling pathways, resulting in PCa cell growth inhibition. The experimental data demonstrated that TCM-1 inhibited EGF/EGFR signaling pathway, and subsequently inhibited both PI3K/AKT and Raf/Erk pathways and their downstream gene expression. Finally, cell growth inhibition and apoptosis were induced by TCM-1 treatment in PCa cells (Fig. 8F).

\section{Discussion}

PCa is one of the most common malignancies worldwide, and is associated with substantial mortality and morbidity (24). In primary PCa, the level of PSA is frequently an important factor in the clinical detection and evaluation of prostate cancer (25). With the further researches on cancer therapy, scientific consensus is that immunotherapy is a promising treatment avenue for cancer (26). Thousands of years of clinical practice in Chinese medicine has suggested that compound Chinese traditional medicines may have unique advantages in treating cancer in the clinic by increasing the ability of the body to
A
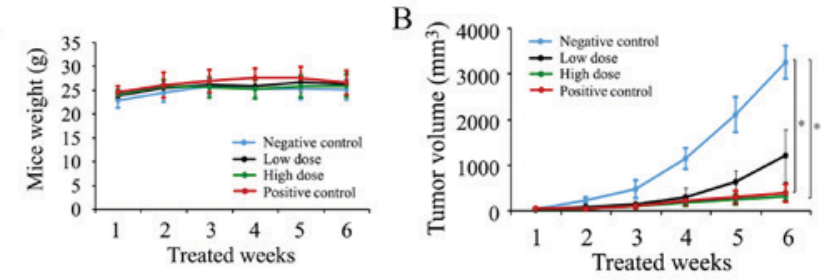

$\mathrm{C}$

D
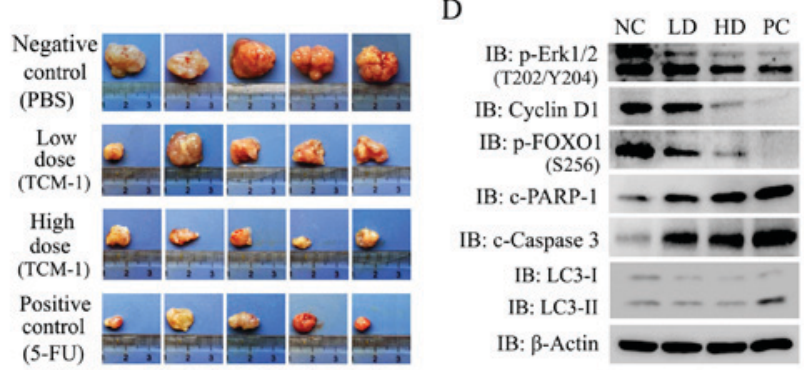

E

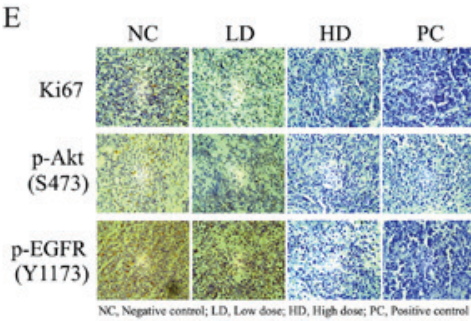

$\mathrm{F}$

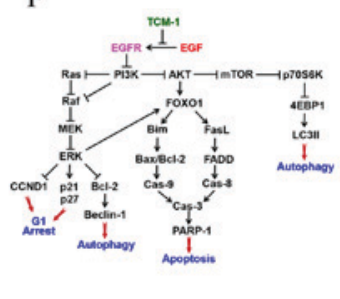

Figure 8. TCM-1 suppresses tumor growth by inhibiting cell growth and inducing cellular autophagy and apoptosis in human PC3 cell xenograft mouse models. PC3 cell tumor xenograft nude mice were treated by oral gavage with TCM-1 and control reagents every 2 days for 6 weeks, and tumor size and mouse weight were monitored at the end of each treatment week. At the end of experiments, the subcutaneous tumors were resected, weighed, photographed and measured immediately. The tumor samples were divided and subjected to an immunohistochemistry assay and western blotting. (A) Mouse weights in the four groups were measured at the end of each treatment week. (B) The average tumor volume of PBS-treated $(n=8)$, TCM-1-treated (including low-dose and high-dose groups; 8 mice/group) and 5-FU-treated $(n=8)$ nude mice at the end of each treatment week. (C) Tumor images from each group of mice at the end of the 6 weeks (five tumors/group are presented). (D) The expression of proteins, including p-AKT Ser473, p-Erk1/2, Cyclin D1, p-FOXO1, c-PARP-1, c-Caspase 3 and LC3-I/II were assayed by western blotting in tumor tissue samples. (E) Immunohistochemistry analysis of the proliferation markerKi-67, p-EGFR (Y1173) and p-AKT(S473) in tumor tissues. (F) Schematic diagram of the apoptotic and autophagy pathways induced by TCM-1 in LNCaP and PC3 cells. "P<0.05. TCM-1, traditional Chinese medicine 1; AKT, RAC- $\alpha$ serine/threonine-protein kinase; Erk, extracellular signal-regulated kinase; FOXO1, forkhead box protein O1; PARP-1, poly(ADP ribose) polymerase 1; c, cleaved; LC3, microtubule-associated proteins 1A/1B light chain 3B; EGFR, epidermal growth factor receptor; $\mathrm{p}$, phosphorylated; 5-FU, 5-fluorouracil.

inhibit and kill cancer cells in vivo, although the underlying molecular mechanisms remain to be elucidated. TCM-1 is a classical compound Chinese traditional medicine which has been used in the clinic to treat patients with prostate cancer in the Jiangsu Hospital of TCM for a number of years. In the present study, it was demonstrated that TCM-1 targeted EGFR and competitively acted on EGFR with EGF, and inhibited the autophosphorylation and activity of EGFR, thereby inhibiting the PI3K/AKT and PI3K/Erk signaling pathways by decreasing the phosphorylation levels of PI3K, AKT and Erk in androgen-dependent and -independent prostate cancer cells (LNCaP and PC3 cells). Inhibition of the PI3K/Erk signaling 
pathway by TCM-1 markedly induced anti-proliferative effects through the induction of cell cycle arrest at the G1 phase in the two cell lines, accompanied by marked alterations in the expression of key cell cycle regulators (Cyclin D1, p21 and p27). Inhibition of the PI3K/AKT signaling pathway by TCM-1 increased FOXO1 transcriptional activity (by decreasing the phosphorylation levels of FOXO1 Ser256/Thr24/Ser319) and induced growth inhibition and initiated cellular apoptosis through the mitochondrial and death receptor pathways in LNCaP and PC3 cells. Additionally, TCM-1-induced inhibition of the PI3K/AKT/mTOR signaling pathway was able to induce cellular autophagy by inhibiting p70S6K and activating LC3-II.

It is known that EGFR and its downstream signaling pathways, including PI3K/AKT/mTOR and Raf/MEK/Erk, serve important roles in a number of types of tissue cell tumorigenesis, and tumor progress and metastasis, and are important in regulating bodily immunity by suppressing $\mathrm{T}$ cell-induced tumor necrosis by decreasing the expression of programmed cell death ligand 1 (PD-L1) $(22,23,27)$. To the best of our knowledge, the present study was the first to demonstrate that TCM-1 targeted EGFR and competitively acted on EGFR with EGF, resulting in auto-phosphorylation and activity inhibition of EGFR, and decreased the activities of the PI3K/AKT and Raf/MEK/Erk pathways. These blockades of EGFR and its downstream signaling pathways may further inhibit the expression of PD-L1 on cancer cell membranes by inhibiting the activity of the mTOR signaling pathway. Therefore, TCM-1 treatment may be associated with the regulation of immunity of patients with prostate cancer. In addition, a number of ligand-receptors on the cell membrane are associated with activation of the PI3K/AKT signal pathway, and promote cancer call growth, proliferation and metastasis (28). Therefore, further studies are required to clearly demonstrate whether EGF/EGFR is the only ligand-receptor involved in the TCM-1-mediated inhibition of the PI3K/AKT signaling pathway, and induction of cell growth inhibition and apoptosis in PCa cells.

The phosphorylation of FOXO1 by AKT inhibits the transcriptional functions of FOXO1, and contributes to cell survival, growth and proliferation $(29,30)$. Conversely, FOXO1 activation has been proposed to be important for regulating apoptosis by stimulating the expression of death receptor ligands, including FasL and TRAIL, in addition to inducing the expression of multiple pro-apoptotic members of the Bcl-2 family (Bim, Bcl-2, BAX). FOXO1 is additionally important for inducing cell cycle arrest via the upregulation of the cell cycle inhibitor p21 and p27 (31,32). The results of the present study demonstrated that TCM-1 decreased the phosphorylation levels of FOXO1 (Ser256/Thr24/Ser319) by inhibiting the PI3K/AKT and Ras/Erk signaling pathways, and induced cell cycle arrest at the G1 phase and cellular apoptosis by inhibiting the expression of Bcl-2/XIAP/survivin, activating Fas-L/Bim/Bax, and increasing the expression of $\mathrm{p} 27$. In addition to FOXO1, FOXO3a is an important member of the FOX family in terms of the regulation of cell growth and apoptosis (30). Therefore, further studies are required to establish whether FOXO3a was additionally involved in TCM-1-induced cell growth inhibition and apoptosis in PCa cells. In a previous study, p53 was indicated to be a key protein in cell growth inhibition and cell apoptosis promotion (33). In the present study, it was observed that the protein level of p53 was unaltered following treatment with TCM-1. It was demonstrated that p53 was not an important factor in TCM-1-induced PCa cell growth inhibition and cell apoptosis.

mTOR is a rapamycin-sensitive serine/threonine protein kinase and serves an important role in regulating cell growth, motility and survival. Dysregulation of the mTOR signaling pathway maybe observed in a number of types of cancer, with PI3K and AKT being upstream regulators of the mTOR signaling pathway (34). Activation of the AKT-mTOR pathway increases the expression of PD-L1 and results in the inactivation of anti-tumor T cells (27). The activation of mTOR complex 1, a principal rapamycin-sensitive mTOR complex, promotes protein synthesis in response to growth factors by increasing the phosphorylation of p70S6K and eukaryotic translation initiation factor 4E-binding protein 1 and exhibits an important role in cellular autophagy (35). According to the present results, TCM-1-induced inhibition of the PI3K/AKT/mTOR signaling pathway impaired the phosphorylation and activity of p70S6K, and resulting in increasing the levels of LC3-II and Beclin-1, which serve essential roles in autophagosome formation $(36,37)$, finally promoting cell autophagy. In the treatment of cancer cell with most anticancer drugs, the cellular processes of autophagy and apoptosis frequently occur simultaneously. Following treatment with TCM-1, it was observed that TCM-1 induced PCa cellular autophagy and apoptosis by inhibiting EGFR and its downstream PI3K/AKT and Raf/Erk pathways. Further studies are required to ascertain whether associations exist between TCM-1-induced PCa cellular autophagy and apoptosis.

TCM has systemic and local therapeutic effects and primarily affects the whole function of the organism. The active components and functional mechanisms are complex; the functional effects of TCM are associated with the active ingredients in each herbal extract. In the study, even though we cannot ensure exactly the same concentration of each component of TCM-1 each time extracting from Chinese herbs, but it's for sure that concentration of each component of TCM-1 in each extraction is almost the same as long as we control the raw herbs.

In conclusion, the present study identified for the first time, to the best of our knowledge, that TCM-1 had the potential to exert a specific anti-cancer effect, in addition to inducing $\mathrm{PCa}$ cell growth inhibition, autophagy and apoptosis in vitro and in vivo, by targeting EGFR and further suppressing the activity of the EGFR/PI3K/AKT and EGFR/PI3K/Erk signaling pathways. The present results may partially provide a molecular basis for the application of TCM-1 in the clinic to treat patients with prostate cancer.

\section{Acknowledgements}

The authors would like to thank Professor Fusong Xu (Jiangsu Province Hospital of Traditional Chinese Medicine, Nanjing, China) for providing the classical prescription of TCM and certain suggestions on the preparation of a water-extract from pharmaceutical raw materials. The present study was supported by grants from the National Natural Science Foundation of China (grant nos. 81272850 and 81472415). 


\section{References}

1. Siegel RL, Miller KD and Jemal A: Cancer statistics, 2015. CA Cancer J Clin 65: 5-29, 2015.

2. Petrylak DP: Chemotherapy for advanced hormone refractory prostate cancer. Urology 54 (Suppl 6A): S30-S35, 1999.

3. Pisters LL: The challenge of locally advanced prostate cancer. Semin Oncol 26: 202-216, 1999.

4. Richie JP: Anti-androgens and other hormonal therapies for prostate cancer. Urology 54 (6A Suppl): 15-18, 1999.

5. Mathew MP, Tan E, Saeui CT, Bovonratwet P, Sklar S, Bhattacharya R and Yarema KJ: Metabolic flx-driven sialylation alters internalization, recycling and drug sensitivity of the epidermal growth factor receptor (EGFR) in SW1990 pancreatic cancer cells. Oncotarget 7: 66491-66511, 2016.

6. De Luca A, Maiello MR, D'Alessio A, Pergameno M and Normanno N: The RAS/RAF/MEK/ERK and the PI3K/AKT signalling pathways: Role in cancer pathogenesis and implications for therapeutic approaches. Expert Opin Ther Targets 16 (Suppl 2): S17-S27, 2012.

7. Brunet A, Datta SR and Greenberg ME: Transcription-dependent and -independent control of neuronal survival by the PI3K-Akt signaling pathway. Curr Opin Neurobiol 11: 297-305, 2001.

8. Yang JY and Hung MC. A new fork for clinical application: Targeting forkhead transcription factorsin cancer. Clin Cancer Res 15: 752-757, 2009.

9. Guo JP, Tian W, Shu S, Xin Y, Shou C and Cheng JQ: IKBKE phosphorylation and inhibition ofFOXO3a: A mechanism of IKBKE oncogenic function. PLoS One 8: e63636, 2013.

10. Coomans de Brachène A and Demoulin JB: FOXO transcription factors in cancer development and therapy. Cell Mol Life Sci 73: $1159-1172,2016$

11. Levine B and Kroemer G: Autophagy in the pathogenesis of disease. Cell 132: 27-42, 2008.

12. Marino G, Niso-Santano M, Baehrecke EH and Kroemer G: Self-consumption: The interplay of autophagy and apoptosis. Nat Rev Mol Cell Biol 15: 81-94, 2014.

13. Yang Y, Ting W, Xiao L, Shufei F, Wangxiao T, Xiaoying W, Xiumei G and Boli Z: Immunoregulation of Shenqi fuzheng injection combined with chemotherapy in cancer patients: A systematic review and meta-analysis. Evid Based Complement Alternat Med 2017: 5121538, 2017.

14. Youns M, Hoheisel JD and Efferth T: Traditional Chinese medicines (TCMs) for molecular targeted therapies of tumours. Curr Drug Discov Technol 7: 37-45, 2010.

15. Sun Y: Application of traditional Chinese medicine in the comprehensive treatment of cancer. Chin J Integr Trad West Med 17: 323-324, 1997 (In Chinese).

16. Li P, Lee H, Guo S, Unterman TG, Jenster G and Bai W: AKT-independent protection of prostate cancer cells from apoptosis mediated through complex formation between the androgen receptor and FKHR. Mol Cell Biol 23: 104-118, 2003.

17. Zhang X, Tang N, Hadden TJ and Rishi AK: Akt, FoxO and regulation of apoptosis. Biochim Biophys Acta 1813: 1978-1986, 2011.

18. Crighton D, Wilkinson S and Ryan KM: DRAM links autophagy to 553 and programmed cell death. Autophagy 3: 72-74, 2007.

19. Lu J, Zhao H, Xu J, Zhang L, Yan L and Shen Z: Elevated cyclin D1 expression is governed by plasma IGF-1 through Ras/Raf/MEK/ERK pathway in rumen epithelium of goats supplying a high metabolizable energy diet. J Anim Physiol Anim Nutr (Berl) 97: 1170-1178, 2013.
20. Heras-Sandoval D, Pérez-Rojas JM, Hernández-Damián J and Pedraza-Chaverri J: The role of PI3K/AKT/mTOR pathway in the modulation of autophagy and the clearance of protein aggregates in neurodegeneration. Cell Signal 26: 2694-2701, 2014.

21. Roskoski R Jr: The ErbB/HER family of protein-tyrosine kinases and cancer. Pharmacol Res 79: 34-74, 2014

22. De Luca A, Carotenuto A, Rachiglio A, Gallo M, Maiello MR, Aldinucci D, Pinto A and Normanno N: The role of the EGFR signaling in tumor microenvironment. J Cell Physiol 214: $559-567,2008$.

23. Howe LR and Brown PH: Targeting the HER/EGFR/ErbB family to prevent breast cancer. Cancer Prev Res (Phila) 4: 1149-1157, 2011

24. Kaplan AL, Hu JC, Morgentaler A, Mulhall JP, Schulman CC and Montorsi F: Testosterone therapy in men with prostate cancer. European Urology 69: 894-903, 2016.

25. Ferro M, Lucarelli G, Bruzzese D, Di Lorenzo G, Perdonà S, Autorino R, Cantiello F, La Rocca R, Busetto GM, Cimmino A, et al: Low serum total testosterone level as a predictor of upstaging and upgrading in low-risk prostate cancer patients meeting the inclusion criteria for active surveillance. Oncotarget 8: 18424-18434, 2017.

26. Burstein HJ, Krilov L, Aragon-Ching JB, Baxter NN, Chiorean EG, Chow WA, De Groot JF, Devine SM, DuBois SG, El-Deiry WS, et al: Clinical cancer advances 2017: Annual report on progress against cancer from the american society of clinical oncology. J Clin Oncol 35: 1341-1367, 2017.

27. Lastwika KJ, Wilson W III, Li OK, Norris J, Xu H, Ghazarian SR, Kitagawa H, Kawabata S, Taube JM, Yao S, et al: Control of PD-L1 expression by oncogenic activation of the AKT-mTOR pathway in non-small cell lung cancer. Cancer Res 76: 227-238, 2016.

28. Westin JR: Status of PI3K/Akt/mTOR pathway inhibitors in lymphoma. Clin Lymphoma Myeloma Leuk 14: 335-342, 2014.

29. Burgering BM and Kops GJ: Cell cycle and death control: Longlive forkheads. Trends Biochem Sci 27: 352-360, 2002.

30. Zhang X, Tang N, Hadden TJ and Rishi AK: Akt, FoxO and regulation of apoptosis. Biochim Biophys Acta 1813: 1978-1986, 2011.

31. Essafi A, Fernández de Mattos S, Hassen YA, Soeiro I, Mufti GJ, Thomas NS, Medema RH and Lam EW: Direct transcriptional regulation of Bim by FoxO3a mediates STI571-induced apoptosis in Bcr-Abl-expressing cells. Oncogene 24: 2317-2329, 2005.

32. Accili D and Arden KC: FoxOs at the crossroads of cellular metabolism, differentiation and transformation. Cell 117: 421-426, 2004.

33. Meek DW: Regulation of the p53 response and its relationship to cancer. Biochem J 469: 325-346, 2015.

34. Lee H, Kim JS and Kim E: Fucoidan from seaweed Fucusvesiculosus inhibits migration and invasion of human lung cancer cell via PI3K-Akt-mTOR pathways. PLoS One 7: e50624, 2012.

35. Bracho-Valdés I, Moreno-Alvarez P, Valencia-Martínez I, Robles-Molina E, Chávez-Vargas L and Vázquez-Prado J: mTORC1-and mTORC2-interacting proteins keep their multifunctional partners focused. IUBMB Life 63: 896-914, 2011.

36. Mizushima N: Methods for monitoring autophagy. Int J Biochem Cell Biol 36: 2491-2502, 2004

37. Guo GF, Jiang WQ, Zhang B, Cai YC, Xu RH, Chen XX, Wang F and Xia LP: Autophagy-related proteins beclin-1 and lc3 predict cetuximab efficacy in advanced colorectal cancer. World J Gastroenterol 17: 4779-4786, 2011. 